\title{
Distributed Computing Architecture using Fog Technology for Improving Intelligent Transportation Systems in Smart City
}

\author{
Zainab H. Ali \\ Lecture, Faculty of Artificial \\ Intelligence, Embedded Network \\ Systems Department, \\ Kafrelsheikh University, Egypt
}

\author{
Shaimaa Hagras \\ Computers' specialist, Faculty of \\ Engineering, Mansoura University, \\ Egypt
}

\author{
Hesham A. Ali \\ Professor, Faculty of Engineering \\ Computer Engineering \& Control \\ Systems Department, Mansoura \\ University, Egypt
}

\begin{abstract}
Intelligent Transportation Systems (ITS) is one of the better Internet of Things (IoT) solutions to fill the gap between IT and automotive activities. Although smart city initiatives have a significant investment of transportation systems to reduce gas emissions and road accidents, the ITS still suffered from several challenges in terms of power consumption and network bandwidth constraint because of the rapid growth in the data dissemination over the vehicular network. The main objective of this study is to present a distributed computing architecture (DCA) based on fog technology for improving transportation services in the smart city. The experimental results show the robustness of the proposed architecture for enhancing vehicular systems in three Quality of Service (QoS) terms is the average energy consumption, packet delivery ratio, and throughput.
\end{abstract}

\section{General Terms}

Intelligent transportation; Internet of Things; Vehicular Adhoc Network; transmission reliability

\section{Keywords}

Ad-hoc Vehicular Network; Fog Technology; Internet of Things; Intelligent Transportation Systems; Quality of Service

\section{INTRODUCTION}

Internet of Things (IoT) [1] has gained great attention in the fields of embedded networks and industrial networking.

IoT can replace human interventions with automated systems such as intelligent healthcare systems, Intelligent Transportation Systems (ITS), environmental monitoring, and intelligent agriculture [1]. These applications use Information and Communication Technology (ICT) to maximize the utilization of existing resources and infrastructure [2]. Although this employment has improved significantly in the IT industries, it may negatively affect network bandwidth and power consumption.

The World Bank announced in 2014 that ITS has consumed around a fifth of total power consumption in the world [3]. To this end, the Cloud Computing (CC) and other state-of-the-art technologies such as Mobile Cloud Computing (MC) and Vehicular Cloud Computing (VC) have provided the successful solutions to increase system capabilities in adjusting the quantity of data transmission over the internet and therefore controlling the rate of power consumption [2]. However, the need for enhancement is still required especially, with the limitations of vehicular network bandwidth.
Fog Computing (FC) is a distributed computing platform that can offer better networking solutions to support locally computing and processing as close as possible to vehicular units. Thus, it can reduce the amount of bandwidth and power used.

The main objective of this study is to present a distributed computing architecture (DCA) using FC technology for improving vehicular networks in smart cities. This proposed architecture affects not only power consumption but also bandwidth usage.

The remainder of this paper is organized as follows: section II reviews the related work. Section III elaborates the proposed DCA based on fog technology. Section IV demonstrates all numerical results. Section V concludes the paper.

\section{RELATED WORK}

Since the early 2000s, the integration between IoT and different cloud computing technology has undergone many academic researches to enhance system scalability. The vehicular networks are one of IoT applications that have used several cloud computing platforms such as $\mathrm{CC}, \mathrm{MC}$, and VC, and $\mathrm{FC}$ for improving their performance. Table 1 shows the different characteristics of these computing platforms.

The recent research directions have been used two technical ways for adjusting the huge amount of data transmission over the vehicular network are: (i) routing techniques and (ii) computing platforms.

Against a backdrop of IoT that has generated an unprecedented volume of composite services and a massive amount of data streaming out in real-time.

Ansari et al.[4] proposed Cloud Computing on Cooperative Cars (C4S) focused on Navigation-as-a-Service (NAVaaS) for constructing Cooperative ITS (C-ITS) that ensures interconnectivity among C-ITS users of single and multiGlobal Navigation Satellite Systems (GNSS) receivers. The architecture that has been suggested enhanced device navigation, but the communication overhead is still limited.

New dynamic architecture and a proof-of-concept system for implementing the computing technology can deal locally with vehicular networks for traffic monitoring and travel time estimation is to fog cloud computing was introduced in [5]. This proposed architecture can improve the overall response time.

Xueshi Hou et al. [6] provided a distributed computing architecture called Vehicular Fog Computing (VFC) to utilize existing vehicular infrastructure via implementing vehicle-as- 
infrastructure. This suggested architecture has high computations and communication capabilities to strength a collaborative multitude of vehicles.

Table 1. Comparative study of CC, MC, VC, and FC technology [2]

\begin{tabular}{|c|c|c|c|c|}
\hline Point of View & $\mathrm{CC}$ & MC & VC & FC \\
\hline $\begin{array}{l}\text { On-demand } \\
\text { services }[4,11]\end{array}$ & $\mathrm{A}$ & $\mathrm{A}$ & $\mathrm{A}$ & A \\
\hline $\begin{array}{l}\text { Elastic } \\
\text { services } \\
{[3,4,11]}\end{array}$ & $\mathrm{A}$ & Possible & Possible & $\mathrm{A}$ \\
\hline $\begin{array}{l}\text { Pay-as-you-go } \\
\text { concept }[4,11]\end{array}$ & $\mathrm{A}$ & $\mathrm{A}$ & $\mathrm{A}$ & $\mathrm{A}$ \\
\hline $\begin{array}{l}\text { Cooperation } \\
{[3,4,11]}\end{array}$ & $\mathrm{A}$ & $\mathrm{A}$ & $\mathrm{A}$ & $\mathrm{A}$ \\
\hline $\begin{array}{l}\text { Location- } \\
\text { awareness } \\
{[4,10,11]}\end{array}$ & N/A & $\mathrm{A}$ & N/A & $\mathrm{A}$ \\
\hline $\begin{array}{l}\text { Data storage } \\
{[3,10,11]}\end{array}$ & High & N/A & Medium & Medium \\
\hline Latency $[4,11]$ & High & No & Medium & $\begin{array}{l}\text { Very } \\
\text { low }\end{array}$ \\
\hline $\begin{array}{l}\text { Bandwidth } \\
\text { usage } \\
{[4,10,11]}\end{array}$ & High & Medium & Medium & $\begin{array}{l}\text { Very } \\
\text { low }\end{array}$ \\
\hline $\begin{array}{l}\text { Power } \\
\text { consumption } \\
{[10,11]}\end{array}$ & High & High & Medium & $\begin{array}{l}\text { Very } \\
\text { low }\end{array}$ \\
\hline $\begin{array}{l}\text { Real-time } \\
\text { response } \\
{[4,10,11]}\end{array}$ & High & Low & Low & $\begin{array}{l}\text { Very } \\
\text { low }\end{array}$ \\
\hline $\begin{array}{l}\text { Management } \\
\text { Model } \\
{[3,4,10,11]}\end{array}$ & $\mathrm{Cz}$. & $\mathrm{Cz}$. & D-Cz. & D-Cz. \\
\hline
\end{tabular}

\section{PROPOSED DISTRIBUTED COMPUTING ARCHITECTURE}

The objective of the proposed DCA is to raise the overall performance of the Vehicular Ad-hoc Networks (VANET), which is considered as the core of the ITS. Recently, academic researchers considered that the massive quantity of data transfer over the Internet as a big challenge in the vehicular networks, which led to maximize the consumption of network bandwidth and power.

The proposed DCA uses the fog technology to control the data transmission rate over the internet and therefore reducing the network bandwidth usage and power consumption alike. As shown in figure 1, the DCA consists of three layers: (i) sensor and registration layer, (ii) the management and fog layer, and (iii) cloud services layer.

In the sensor and registration layer, IoT devices such as smart vehicles, communication vehicle units, base station, RoadSide Units (RSU), and On-Board Unit (OBU) are connected to each other by exchange their information. Each IoT device can connect with fog layer through a message to ask it about traffic services.

The fog nodes in the DCA divided into two types of nodes: Fog Brokers (FB) and Fog Servers (FS). In the sensor and registration layer, each $\mathrm{FB}$ covers a specific vehicular area. This area is determined by its communication range of FB for ensuring network connectivity. Assume that the communication ranges of every FBs are equal. The FB send periodically broadcast message contains its ID and its registration area $\mathrm{ID}$. Since the vehicles receive this message, they can register themselves at the FB.

In the management and fog layer, each FB asks the vehicles about their information to build its local database. These databases shared between FB nodes and FS for facilitating navigation processing and mitigate the need to access CC continuously. By default, the FB services are available only for the registered vehicles. The requests among vehicles handled by FBs and the requests among FBs handled by FS. This procedure helps to reduce the volume of data transmission over the CC.

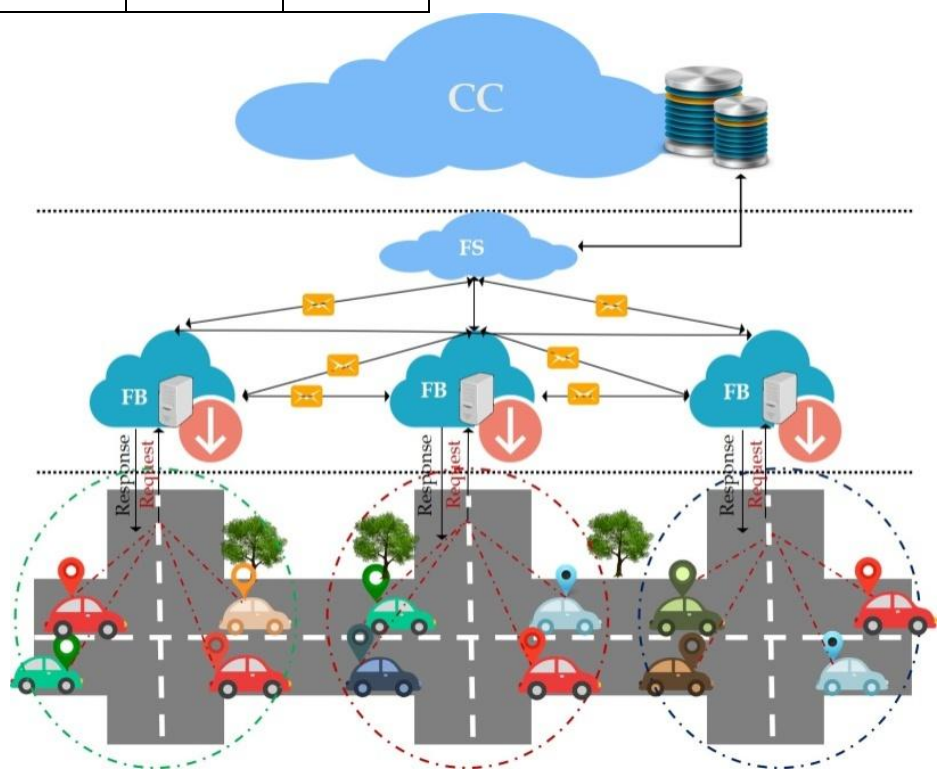

Fig 1: The proposed DCA based on fog technology 
In the cloud services layer, the global overview about vehicular network sends via the FS to the CC. Basically, the $\mathrm{FS}$ only can send requests to the $\mathrm{CC}$ for reducing the rate of the communication traffics between vehicular units and CC. The FS sends requests for the $\mathrm{CC}$ in case there is no response about a specific vehicle or in case there is a need for higher processing. The main steps of the proposed DCA are illustrated in flowchart in fig 2 .

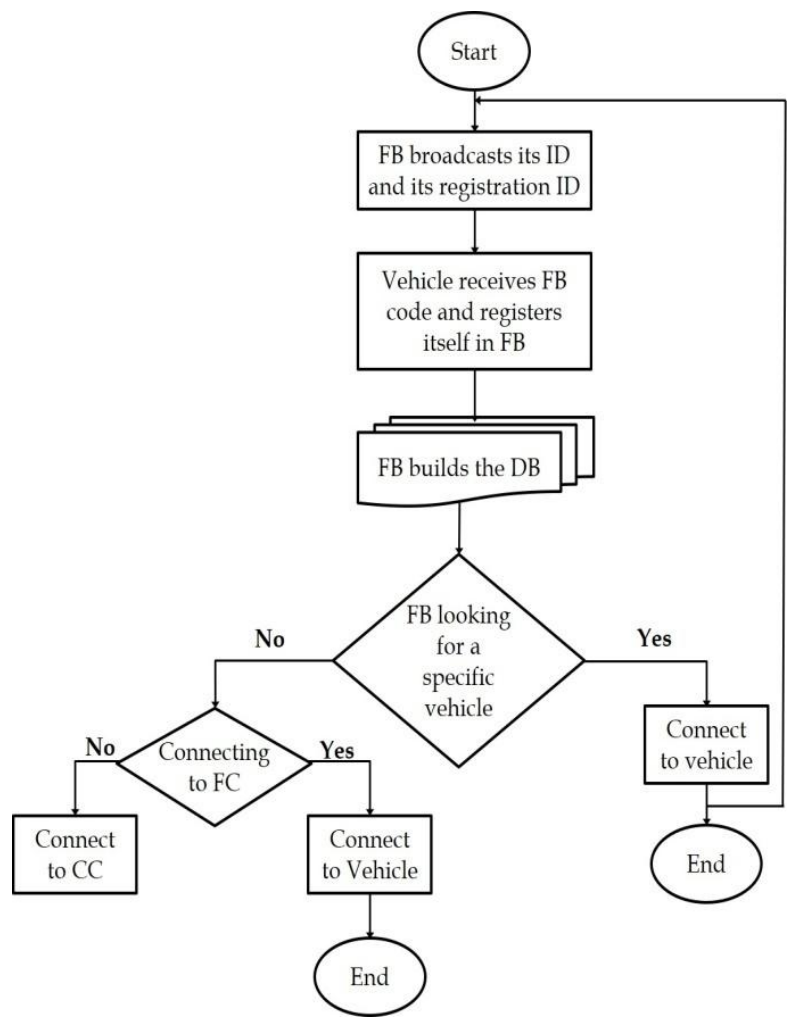

Fig 2: The flowchart of the main steps in the proposed DCA

\section{NUMERICAL RESULTS}

The experimental settings have been conducted using a desktop application with Intel Core i7-6500U (dual-core, $4 \mathrm{MB}$ cache, up to $3.1 \mathrm{GHz}$ with Turbo Boost), Intel HD Graphics 520, 12GB LPDDR3 SDRAM $(1,866 \mathrm{MHz})$, and a 64-bit PC (AMD64) Ubuntu operating system. Network Simulation (NS2.35) has been used as the simulation tool to design network topology. Fog nodes were simulated according to the reference guide known Fog Hierarchical Deployment Model from Open Fog Reference Architecture [7]. Table 2 depicts the all-network setting.

The proposed architecture was compared against a set of state-of-the-art techniques in $[8,9,10]$ and [11] in order to prove the worthiness of our proposal in terms of power and network bandwidth consumption. The power consumption was used as a QoS performance metric to measure the rate of energy depletion in the vehicle units. As seen in fig. 3, the highest rate of energy depletion in the term of data transmission was obtained by the techniques in [9] and [11]; that is because the data generated by smart vehicles need constantly traveling to cloud computing for processing. This process consumes sensors' battery, and it adversely affects the network lifetime. The lowest rate of power consumption rate was recorded by both the proposed architecture and direct transmission schema. The reason was that FC used to apply the energy-aware load distribution and therefore the data is locally processing without the need to send it to the cloud server. The TPEG [10] demonstrated a little difference between the proposed architecture as well as it ranked the second level.

Table 2. Parameters of the simulation and network settings

\begin{tabular}{|l|l|}
\hline Parameters & \multicolumn{1}{|c|}{ value } \\
\hline Network Range & 10 to100 \\
\hline Channel Type & Wireless \\
\hline Packet size & 512 bytes \\
\hline Traffic Source & CBR \\
\hline Number of seed & 1 \\
\hline Traffic agent & Node-UDP \\
\hline Routing Protocol & AOMDV \\
\hline MAC protocol & IEEE $802.11 \mathrm{p}$ \\
\hline Time & 1000 Sec \\
\hline Antenna Model & Omni \\
\hline Energy Model & Battery \\
\hline
\end{tabular}

The proposed architecture was compared against a set of techniques in $[8,9,10]$ and $[11]$ to show the efficacy of our proposal. The consumption of the energy was deployed as a QoS metric to measure the rate of energy depletion in the vehicular network, as seen in fig. 3. The highest rate of energy depletion in data transfer was obtained by the techniques in [8] and [11]. While the proposed architecture was ranked at a low rate of energy consumption because of applying the energy-aware load distribution of FC. The TPEG [10] demonstrated a little difference between the proposed architecture as well as it ranked the second level.

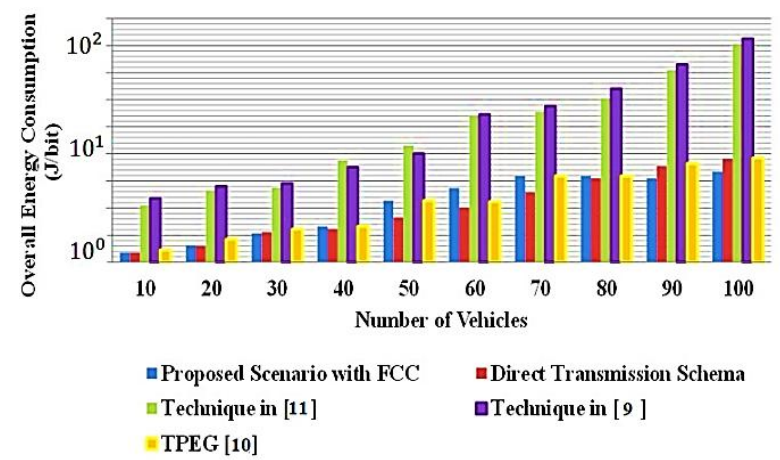

Fig 3: The average energy consumption vs. the number of vehicles

From the network point of view, the size of the received packet highly affects bandwidth and overall network performance. This experiment evaluates the efficiency of the proposed architecture in term of packet delivery ratio. As shown in figure 4 , the proposed architecture was compared 
with other techniques in [8], [9], and [11] in order to measure its performance in saving network bandwidth with a successful rate of delivered messages. The first place in packet delivery rates was the proposed architecture that is because fog technology increases reliability in data packet transmission. Moreover, the FC can efficaciously meet the demands for traffic congestion as well as it can achieve faster analysis of big data in real-time. The technique in [9] and [11] came in second place. The lowest rate of data packet delivery was to direct transmission schema.

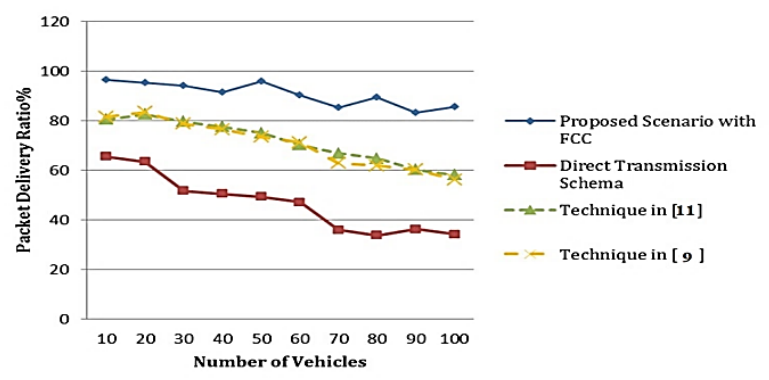

Fig 4: The packet delivery ratio vs. the number of vehicles

Another QoS metric to measure network bandwidth usage and its performance, that is throughput. Surly, the data transmission rate is influenced by many subjective factors of the network such as link quality over the internet, bandwidth, and network throughput. On the other hand, achieving a high data transmission rate is heavily related to the packet delivery ratio. Figure 5 depicts the comparison between the proposed architecture and a set of recent techniques to prove the efficiency of our scenario in term of network throughput. Both proposed architecture base on the fog technology and direct transmission schema ware attached the highest point, this is a result of the fog technology can process data locally without the need to use transfer it to a cloud server. Therefore, the network bandwidth is reduced, and overall network performance is enhanced. The second place was achieved by TPEG [10]. We observe that techniques in [9] and [11] in term of throughput have a wobbly performance with increasing the average vehicles' speed, and they were ranked the lowest point in saving the vehicular network bandwidth.

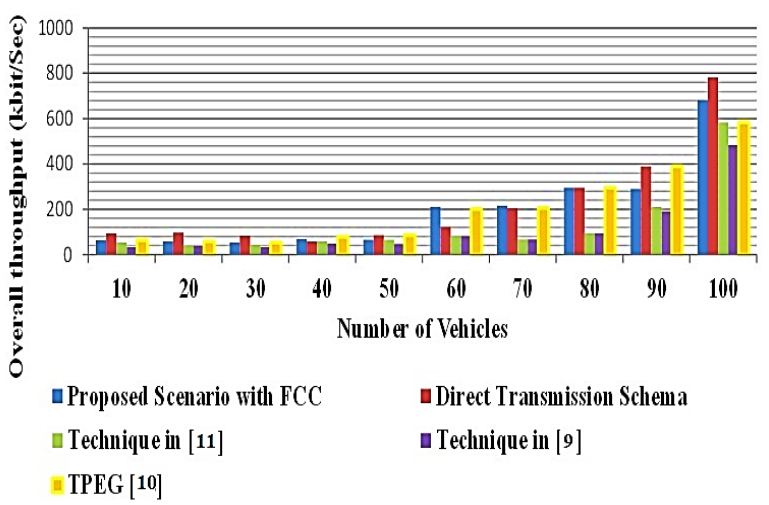

Fig 5: The overall throughput vs. the number of vehicles

\section{CONCLUSION AND FUTURE WORK}

IoT enables the growth in real-time systems and applications such as ITS, smart cities, and intelligent healthcare systems. In 2014, the World Bank report announced that the ITS consumed around a fifth of total energy in the world. From the QoS point of view, the huge amount of data transfer plays a key role in increasing the consumption of network bandwidth usage and energy alike. This paper introduced a new architecture called DCA utilizing fog technology to reduce the communication traffics between vehicular units and CC. Hence, the massive amount of data transmission is also reduced. The improvement in terms of network connectivity and transmission reliability are still the hot topics in the ITS, and thus it is considered a significant point in future work.

\section{REFERENCES}

[1] Zainab H. Ali, Hesham H. Arafat, Mahmoud Badawy. "Internet of Things (IoT): definitions, challenges and recent research directions". International Journal of Computer Applications. Vol: 128, pp 37-47, 2015.

[2] Zainab H Ali, Mahmoud M Badawy, Hesham A Ali. "A novel geographically distributed architecture based on fog technology for improving Vehicular Ad hoc Network (VANET) performance". Peer-to-Peer Networking and Applications, Springer US. pp 1-28. (2020). DOI https://doi.org/10.1007/s12083-020-00910-9.

[3] Ali, Z.H., Ali, H.A. "Towards sustainable smart IoT applications architectural elements and design: opportunities, challenges, and open directions". J Supercomput $\quad 77, \quad 5668-5725 \quad$ (2021). https://doi.org/10.1007/s11227-020-03477-7

[4] K. Ansari, in 2018 IEEE 11th International Conference on Cloud Computing (CLOUD), pp. 794-801. (2018).

[5] Pereira, L. Ricardo, M. Lu'1s, C. Senna, S. Sargento. "Assessing the reliability of fog computing for smart mobility applications in vanets". Future Generation Computer Systems 94, 317 (2019).

[6] X. Hou, Y. Li, M. Chen, D. Wu, D. Jin, S. Chen. "Vehicular fog computing: A viewpoint of vehicles as the infrastructures". IEEE Transactions on Vehicular Technology 65(6), 3860 (2016).

[7] Openfog reference architecture for fog computing.https://www.openfogconsortium.org/. Accessed: 2020-7-26.

[8] A.A. Alahmadi, A.Q. Lawey, T.E. El-Gorashi, J.M. Elmirghani, "in Network of the Future (NOF)". 2017 8th International Conference on th (IEEE, 2017). pp. 22-26. (2017).

[9] R.A. Osman, X.H. Peng, M. Omar. "Adaptive cooperative communications for enhancing qos in vehicular networks", Physical Communication. (2018).

[10] Y. Lai, F. Yang, J. Su, Q. Zhou, T. Wang, L. Zhang, Y. $\mathrm{Xu}$. "Fog-based two-phase event monitoring and data gathering in vehicular sensor networks". Sensors 18(1), 82 (2018).

[11] Y. Li, A. Orgerie, I. Rodero, B.L. Amersho, M. Parashar, J.M. Menaud. "End-to-end energy models for Edge Cloud-based IoT platforms: Application to data stream analysis in IoT". Future Generation Computer Systems (2017). DOI https://doi.org/10.1016/j.future.2017. 